\title{
Facilitative and inhibitory effects of cuing sound duration, intensity, and timbre
}

\author{
TODD A. MONDOR \\ University of Manitoba, Winnipeg, Manitoba, Canada \\ and \\ TODD E. LACEY \\ Mount Allison University, Sackville, New Brunswick, Canada
}

\begin{abstract}
Two experiments are reported in which the possibility that auditory attention may be controlled in a stimulus-driven manner by duration, intensity, and timbre cues was examined. In both experiments, listeners were presented with a cue followed, after a variable time period of a 150-, 450-, or 750-msec stimulus onset asynchrony (SOA), by a target. In three different conditions for each experiment, the duration, intensity, or timbre relation between the cue and the target was varied so that, on $50 \%$ of the trials, the two sounds were identical and, on $50 \%$ of the trials, the two sounds were different in the manipulated feature. The two experiments differed only in the judgment required, with listeners in Experiment 1 identifying the duration, intensity, or timbre of the target and listeners in Experiment 2 indicating whether the target incorporated a brief silent gap. In both experiments, performance was observed to depend on both the similarity of and the time between the cue and the target. Specifically, whereas at the 150-msec SOA performance was best when the target was identical to the preceding cue, at the 750-msec SOA performance was best when the cue and the target differed. This pattern establishes the existence of duration-, intensity-, and timbre-based auditory inhibition of return. The theoretical implications of these results are considered.
\end{abstract}

There is a long history to the experimental investigation of auditory selective attention (e.g., Cherry, 1953; Moray, 1972). Much of this research, particularly that accomplished in the 1950s, 1960s, and 1970s, examined the ability of listeners to selectively attend to one verbal message while, at the same time, ignoring a competing message (e.g., Moray, 1972; Treisman, 1960, 1972). Although it appears that a variety of perceptual and semantic characteristics may be used to direct attention voluntarily, there emerged a general agreement that basic acoustic features, such as frequency and location, were most salient in facilitating selection of a desired message (e.g., Broadbent, 1957, 1958; Johnston \& Heinz, 1978). Far from being entirely controlled by characteristics of the message itself, however, many investigators demonstrated clearly that listening strategies were important as well, particularly with regard to switching attention between competing channels (see Moray, 1972, for a discussion).

Investigation of auditory attention has not been confined to studies of the selection of verbal information. On the contrary, a tradition of examining the selection of

This research was supported by a grant from the Natural Sciences and Engineering Research Council of Canada to T.A.M. We thank Nicole Terrio for her invaluable assistance in collecting and analyzing data. Correspondence concerning this article should be addressed to T. A. Monder, Department of Psychology, Duff Roblin Bldg., University of Manitoba, Winnipeg, MB R3T 2N2, Canada (e-mail: todd_mondor@ umanitoba.ca). nonverbal sounds also developed throughout this period. For example, a great deal of research has been performed to delineate sensitivity to quiet or brief auditory signals (e.g., Carterette, Friedman, \& Wyman, 1966; Creelman, 1959; Green, McKey, \& Licklider, 1959; Jeffress, 1967). Results obtained from many such experiments have established that the accuracy of detection of a pure tone embedded in noise (this sound is usually referred to as the probe) is best when the frequency expected by the listener matches the frequency of the sound actually presented, with performance declining as the frequency difference between the probe and expectation increases (e.g., Dai, Scharf, \& Buus, 1991; Huggins, 1952; Johnson \& Hafter, 1980; Macmillan \& Schwartz, 1975; Penner, 1972; Scharf, Quigley, Aoki, Peachey, \& Reeves, 1987; Schlauch \& Hafter, 1991; Sorkin, Pastore, \& Gilliom, 1968). Although, this frequency sensitivity effect has been generally regarded as the result of a low-level perceptual process involving evaluation of the output of one or more auditory filters (e.g., Creelman, 1959; Green et al., 1959), central cognitive factors have also been identified as important (e.g., Greenberg \& Larkin, 1968; Swets, 1963). Of course, such an interpretation could easily be incorporated into an attentional account in which the frequency sensitivity effect is viewed as the expression of underlying voluntary attentional processes established through the listeners' expectations (see Mondor \& Bregman, 1994). 
Thus, although more empirical investigation of visual selective attention has been accomplished in recent decades, important discoveries have been made regarding the nature and characteristics of the attentional processes devoted to the selection of auditory information. By and large, however, these discoveries have related to the voluntary control of attentional processes (e.g., Moray, 1974; Moray, Fitter, Ostry, Favreau, \& Nagy, 1976; Treisman, 1969). More recently, the operation of auditory selective attention has also been investigated using paradigms in which voluntary or strategic control is thought to exert little influence on performance. In one of these paradigms, listeners are presented with two sounds in succession, and either a detection or a discrimination response is required to the second of these (this second sound is usually referred to as the target). These experiments are intended to identify the conditions under which the first sound (this sound is usually referred to as the cue) influences the speed and accuracy with which a response can be made to the target. The now firmly established finding is that target judgments depend on the joint effects of cue-target similarity in the basic acoustic features of location and frequency, as well as on the time period between the onsets of the cue and the target (stimulus onset asynchrony, SOA). Specifically, whereas at brief SOAs of about $100 \mathrm{msec}$, performance is better when the target sounds from the same location or is of the same frequency as the preceding cue, at longer SOAs of about $700 \mathrm{msec}$, performance is best when the target and the cue differ (e.g., Mondor, Breau, \& Milliken, 1998; Reuter-Lorenz, Jha, \& Rosenquist, 1996; Schmidt, 1996; Spence \& Driver, 1998). Because this performance pattern is obtained when there is no predictive relation between the cue and the target (i.e., the cue and target are as likely to differ as they are to be the same in frequency or location), it appears that the cue itself acts to control attention. Such stimulus-driven control over attention appears to occur independently of the voluntary intent of the listener.

A transition from an advantage for location repetition at brief SOAs to an advantage for change at more lengthy SOAs has been reported in studies of visual covert orienting, where it has been labeled inhibition of return, or IOR (e.g., Maylor \& Hockey, 1985; Posner \& Cohen, 1984; Rafal, Calabressi, Brennan, \& Sciolto, 1989; Tipper, Weaver, Jerreat, \& Burak, 1994). Thus, the studies of auditory covert orienting reviewed above document the existence of both location-based and frequency-based auditory IOR. ${ }^{1}$ Furthermore, and again following the visual attention literature, the advantage for a repetition of location or frequency apparent at brief SOAs is often referred to as a facilitative effect, and the advantage for a change in location or frequency at more lengthy SOAs is usually labeled an inhibitory effect.

Investigations of stimulus-driven control of auditory covert orienting have concentrated on the effects on target judgments of advance information regarding frequency or location in the form of a cue. This initial interest in the possibility that location and frequency information may be used to guide selection is quite consistent with the notion that these features are, in many situations, perceptually dominant over other basic acoustic features (e.g., Bregman, 1990). Although there is no doubt that frequency and location are fundamentally important acoustic attributes, other elementary features, such as duration, intensity, and timbre, may also be used successfully to discriminate sounds. To the extent that there is a connection between the perceptual basis for differentiating sounds and the features that may be used to control attention, it is possible that sound selection may be guided by advance duration, intensity, and timbre information. The experiments reported below were designed to evaluate this possibility. To anticipate somewhat, all three of these secondary perceptual features are shown to generate both facilitative and inhibitory effects on target judgments.

\section{EXPERIMENT 1}

Performance was examined in three separate conditions in Experiment 1, all of which were founded on the cue-target paradigm discussed above. In the duration condition, the cue and the target either were identical (i.e., same location, frequency, duration, intensity, and timbre) or differed only in duration (50 vs. $100 \mathrm{msec}$ ). Listeners were required to judge the duration of the target (brief or long). The intensity and timbre conditions were similar to the duration condition, except that the manipulated and the judged features in these conditions were intensity and timbre, respectively, rather than duration.

\section{Method}

\section{Subjects}

Eighty-four undergraduate students attending Mount Allison University voluntarily participated in exchange for course credit. Twentyeight different listeners took part in each of the duration, intensity, and timbre conditions. None of the participants reported any hearing impairment.

\section{Materials}

Computer and sound system. The experiment was controlled by a 486/50 PC running the MAPLE programming environment (Bregman, Achim, \& Ahad, 1992). Sounds were presented over an AcousticProfiles speaker positioned directly in front of each listener at $0^{\circ}$ azimuth.

Sounds. All sounds were synthesized at $32000 \mathrm{~Hz}$, using the MITSYN signal-processing software package (Henke, 1990), with 5-msec onset and offset amplitude ramps to eliminate onset and offset clicks. For the duration condition, two pure tones of $500 \mathrm{~Hz}$ each were synthesized, one with a duration of $50 \mathrm{msec}$ and the other with a duration of $100 \mathrm{msec}$. The intensities at which the brief and the long sounds were presented was adjusted so that they were the same subjective intensity. For the intensity condition, two pure tones of $500 \mathrm{~Hz}$ and 50-msec duration each were synthesized, one at $80 \mathrm{~dB}$ SPL, the other at $65 \mathrm{~dB}$ SPL. For the timbre condition, two complex sounds of 50-msec duration were generated. One complex sound was composed of 500 and $1000 \mathrm{~Hz}$ components, with the $1000-\mathrm{Hz}$ component half the intensity of the $500-\mathrm{Hz}$ component. The second 
complex sound was synthesized with the same frequency components, but the $500-\mathrm{Hz}$ component was half the intensity of the 1000 $\mathrm{Hz}$ component. These two sounds were arbitrarily labeled Sound A and Sound B, respectively. The sounds were of equivalent subjective intensity and were presented at approximately $65 \mathrm{~dB}$ SPL. Thus, the experimental manipulation of timbre was equated with a manipulation of the relative intensities of the frequency components that made up each sound (see, e.g., Handel, 1989, Hirsh \& Watson, 1996, Iverson \& Krumhansl, 1993, and Moore, 1989, who have argued that timbre may be so defined).

\section{Design and Procedure}

Different groups of 28 volunteers participated in three different experimental conditions. In all the conditions, each trial consisted of two sounds presented in succession. On any particular trial, the first sound (i.e., the cue) and the second sound (i.e., the target) could be either the same or different in the manipulated feature. (For explanatory convenience, a trial on which the target repeats the duration, intensity, or timbre of the cue will be referred to as a repeat trial, and a trial on which the target differs from the cue will be referred to as a change trial.) Across all the trials within a condition, the probability that the cue and the target would be identical on the variable of interest was set to .5. The time period between the onset of the cue and the onset of the target was equally likely to be 150 , 450 , or $750 \mathrm{msec}$ (SOA).

All responses were made using the 1 (brief duration, quiet, and Sound A) and the 0 (long duration, loud, and Sound B) keys on a computer keyboard. These stimulus-response mappings were reversed for half of the listeners in each condition. For each condition, the listeners performed 36 practice trials ( 6 for each combination of trial type and SOA), followed by 144 experimental trials (24 for each combination of trial type and SOA). The experiment was selfpaced in that the participants pressed any key on the keyboard to initiate each trial. Each trial began $750 \mathrm{msec}$ following this initiating keypress. The participants were encouraged to respond as quickly and accurately as possible. Response times (RTs) were measured from the onset of sounds in the intensity and timbre conditions and from the offset of sounds in the duration condition. ${ }^{2}$ Statistical analysis of RT was performed using only correct responses. RTs more than 2.5 standard deviations from the mean were excluded as outliers.

\section{Results}

As is shown in Table 1 and Figure 1 (left panel), the obtained results revealed a consistent pattern of performance in all three conditions. Specifically, whereas at the 150msec SOA performance was better if the target was the same duration, intensity, or timbre as the cue (i.e., a facilitative effect), at the 750-msec SOA performance was better when the target differed from the cue (i.e., an inhibitory effect). This transition in the influence of an uninformative cue from facilitation to inhibition as SOA increases provides the first evidence of the existence of duration-, intensity-, and timbre-based auditory IOR.

Statistical examination of RT and percentage of errors was performed separately for each of the duration, intensity, and timbre conditions, using two-way withinsubjects analyses of variance (ANOVAs), with SOA (150, 450 , or $750 \mathrm{msec}$ ) and trial type (repeat or change) acting as independent variables. Although the results obtained from these analyses were virtually identical, performance in each condition is discussed separately below for clarity, followed by a comparison of performance in the three conditions.

\section{Duration}

The analysis of performance in the duration condition revealed significant main effects of SOA $[F(2,54)=$ $52.78, p<.001]$ and trial type $[F(1,27)=3.93, p=.055]$ and a significant interaction between the two variables $[F(2,54)=15.65, p<.001]$. The significant interaction was obtained because, whereas performance for the repeat trials was better than performance for the change trials at the $150-$ msec SOA $(p<.001)$, the reverse was apparent at the $750-\mathrm{msec}$ SOA $(p<.01)$.

The analysis of the error data showed that main effects of SOA $[F(2,54)=74.50, p<.001]$ and trial type $[F(1,27)=20.76, p<.001]$ were significant. In addition, an SOA $\times$ trial type interaction $[F(2,54)=16.91, p<$ $.001]$ arose because an advantage for the repeat trials at the $150-$ msec SOA $(p<.001)$ was eliminated at SOAs of 450 and $750 \mathrm{msec}(p>.10$ in both cases).

\section{Intensity}

The ANOVA of the RT data revealed significant main effects of SOA $[F(2,54)=110.90, p<.001]$ and trial type $[F(1,27)=28.82, p<.001]$. Generally, performance was better on the repeat trials and improved as SOA increased. More important, however, the SOA $\times$ trial type interaction was also significant $[F(2,54)=$ $35.23, p<.001]$. Planned comparisons indicated that this interaction arose because an advantage for the repeat tri-

Table 1

Response Times (RTs), Percentage of Errors (PE), and Standard Errors for the RT Data as a Function of Cue-Target Relation (Duration, Intensity, or Timbre), Trial Type (Repeat or Change), and Stimulus Onset Asynchrony $(150,450$, or $750 \mathrm{msec})$ in Experiment 1

\begin{tabular}{|c|c|c|c|c|c|c|c|c|c|c|}
\hline \multirow{3}{*}{$\begin{array}{c}\text { Cue-Target } \\
\text { Relation }\end{array}$} & \multirow{3}{*}{$\begin{array}{l}\text { Trial } \\
\text { Type }\end{array}$} & \multicolumn{9}{|c|}{ Stimulus Onset Asynchrony } \\
\hline & & \multicolumn{3}{|c|}{$150 \mathrm{msec}$} & \multicolumn{3}{|c|}{$450 \mathrm{msec}$} & \multicolumn{3}{|c|}{$750 \mathrm{msec}$} \\
\hline & & RT & $\mathrm{PE}$ & $S E$ & RT & $\mathrm{PE}$ & $S E$ & RT & $\mathrm{PE}$ & $S E$ \\
\hline \multirow[t]{2}{*}{ Duration } & Repeat & 653 & 23.1 & 23.87 & 559 & 11.2 & 18.92 & 555 & 9.9 & 20.27 \\
\hline & Change & 721 & 34.1 & 30.06 & 564 & 16.2 & 18.76 & 528 & 8.4 & 18.92 \\
\hline \multirow[t]{2}{*}{ Intensity } & Repeat & 653 & 9.2 & 25.02 & 598 & 8.0 & 21.3 & 587 & 9.2 & 21.68 \\
\hline & Change & 830 & 31.3 & 33.67 & 613 & 8.9 & 21.42 & 546 & 9.2 & 23.84 \\
\hline \multirow[t]{2}{*}{ Timbre } & Repeat & 604 & 11.6 & 18.36 & 576 & 12.5 & 18.41 & 559 & 11.3 & 17.19 \\
\hline & Change & 724 & 16.7 & 25.48 & 586 & 12.4 & 19.18 & 541 & 12.8 & 17.39 \\
\hline
\end{tabular}




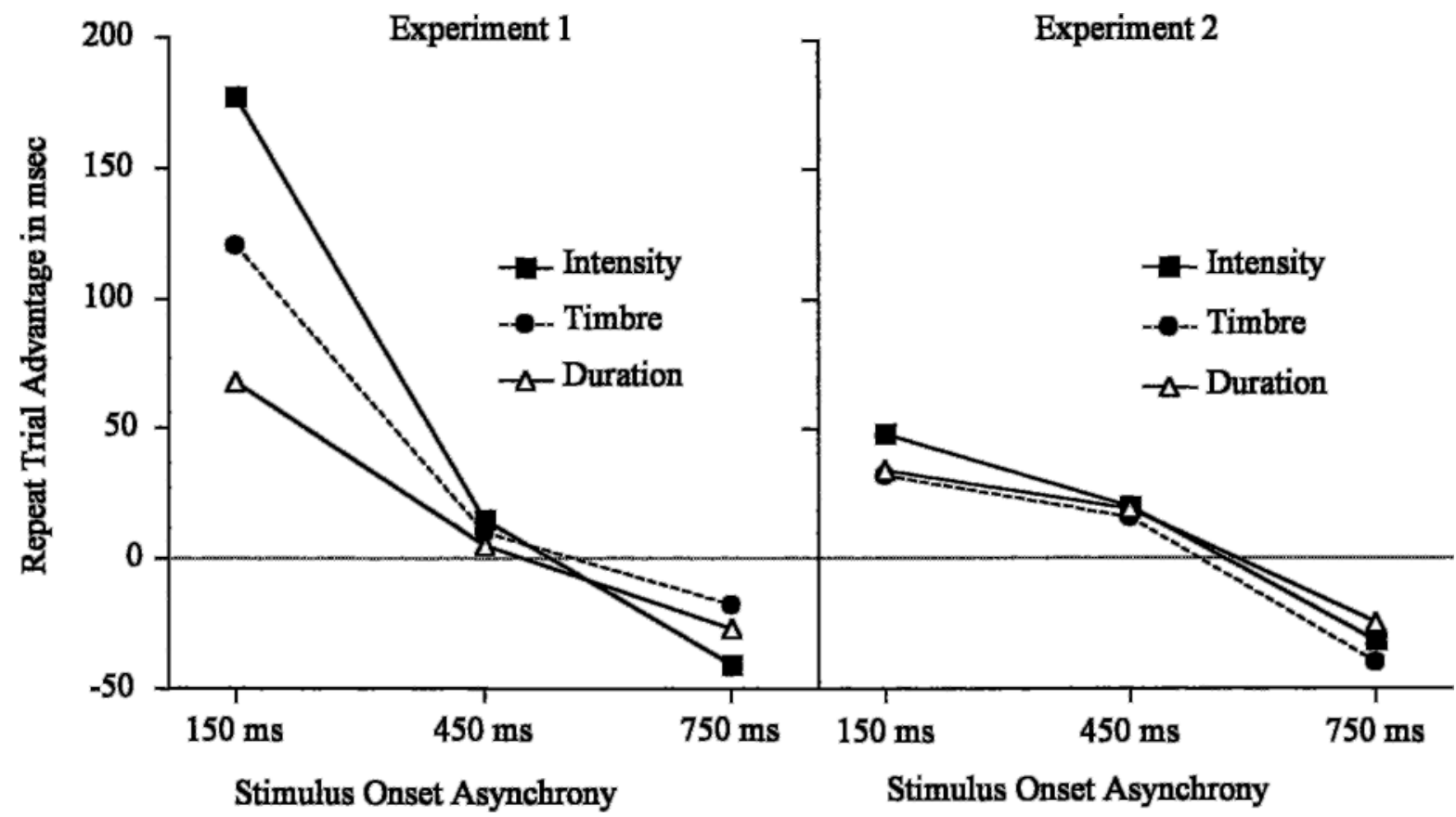

Figure 1. Repeat trial advantage in milliseconds as a function of stimulus onset asynchrony and condition in Experiments 1 and 2.

als at the $150-\mathrm{msec}$ SOA $(p<.001)$ reversed to an advantage for the change trials at the 750-msec SOA $(p<.01)$.

The analysis of the error data indicated significant main effects of SOA $[F(2,54)=39.87, p<.001]$ and trial type $[F(1,27)=46.30, p<.001]$, as well as a significant interaction between the two variables $[F(2,54)=62.82$, $p<.001]$. Further exploration of the interaction revealed a significant advantage for the repeat trails at the 150$\operatorname{msec}(p<.001)$ and 450-msec SOAs $(p<.05)$, but no difference between trial types was apparent at the 750msec SOA.

\section{Timbre}

A statistical analysis of the RT data identified significant main effects of trial type $[F(1,27)=27.50, p<.001]$ and SOA $[F(2,54)=62.84, p<.001]$, as well as a significant interaction between trial type and $\mathrm{SOA}[F(2,54)=$ $37.77, p<.001]$. As was the case for the duration and intensity conditions, planned comparisons verified that, in comparison with performance on change trials, performance on the repeat trials was significantly better at the $150-\mathrm{msec}$ SOA $(p<.001)$ but significantly worse at the $750-\mathrm{msec}$ SOA $(p<.05)$

A complementary analysis of percentage of errors revealed that although the main effect of trial type was significant $[F(1,27)=6.28, p<.02]$, the main effect of SOA was not $[F(2,54)=1.86, p=.16]$. However, a significant trial type $\times$ SOA interaction $[F(2,54)=5.20, p<$ $.01]$ emerged, because an advantage for the repeat trials at the $150-\mathrm{msec}$ SOA $(p<.01)$ was eliminated at the $450-$ and 750-msec SOAs ( $p>.1$ in both cases).

\section{Comparison of Performance in the Duration, Intensity, and Timbre Conditions}

A three-way mixed ANOVA (condition [duration, intensity, or timbre $] \times$ SOA $[150,450$, or $750 \mathrm{msec}] \times$ trial type [repeat or change]) was performed, using RT as the dependent variable, to determine whether performance differed substantially between conditions. As is suggested by an inspection of Figure 1 (left panel), there was a significant interaction between condition, SOA, and trial type $[F(4,162)=5.15, p<.01]$. Further analysis revealed that this interaction arose because of a significantly larger facilitative effect in the intensity condition than in either the timbre $(p<.05)$ or the duration $(p<.001)$ condition and a smaller facilitative effect in the duration condition than in the timbre condition $(p<.05)$. The three conditions did not differ in the magnitude of the performance difference between the repeat and the change trials at either the 450 - or the $750-\mathrm{msec}$ SOA ( $p>.40$ at both SOAs).

A complementary analysis of the error data revealed similar results. The three-way interaction reached statistical significance $[F(4,162)=7.65, p<.001]$ because of a larger facilitative effect for the intensity condition than for either of the other conditions $(p<.05$ for both comparisons). Although the facilitative effect for the duration condition appeared to be larger than that for the timbre condition, this difference was not significant $(p=.18)$.

The reason for the differences in the magnitude of facilitation effects for the three conditions is not clear. It is possible that the differences simply reflect random variation owing to the use of a between-subjects design. Alternatively, the differences may reflect an underlying in- 
equality in the degree to which each of the three features engages attentional processes. If this latter possibility is correct, these asymmetries in the magnitude of the facilitative effects should be replicated in Experiment 2, in which a judgment orthogonal to the manipulation of cuetarget similarity is required.

\section{Discussion}

These results establish that the perceptual attributes of duration, intensity, and timbre may act individually to guide auditory attention. Moreover, the transition from an advantage for the repeat trials at the 150-msec SOA to an advantage for the change trials at the 750-msec SOA establishes the same performance function as that previously reported in studies in which frequency and location cues were used to control attention (e.g., Mondor \& Breau, 1999; Mondor et al., 1998). Although it would appear that these effects are produced through an influence of the independent variables on attentional processes, other explanations are also possible. In particular, performance may have been significantly influenced by the fact that the response required of listeners was directly related to the manipulation of cue-target similarity. It is certainly possible that, under such circumstances, the information provided by the cue might engender a tendency or preference to respond with one of the two alternatives. Indeed, such nonattentional effects have been identified previously as constituting serious impediments to accurate interpretation of the extent to which advance cues engage attentional processes (see, e.g., Carterette et al., 1966, Greenberg \& Larkin, 1968, Kinchla, 1992, and Shaw, 1978, 1984, for discussions of this issue). Of course, to account for the performance pattern apparent in Experiment 1, the direction of such a bias would have to vary as a function of SOA, with an initial bias toward executing a response consistent with the information provided by the cue reversing to a bias toward executing a response inconsistent with it. One strategy that has been used previously to eliminate potential confounds based on such responses biases has been to require judgments that are orthogonal to the manipulation of cue-target similarity. The rationale on which this approach is based is that because the cue provides no information about the required response, any effect of its presentation on performance cannot be attributed to response biases. For example, in an examination of the effect of advance frequency and location cues on judgments of a subsequent target, Mondor and Breau (1999) required listeners to identify the rise-time of the target (fast vs. slow). Their study confirmed the earlier finding by Mondor et al. (1998) that both types of cues act to produce facilitative effects at brief SOAs and inhibitory effects at long SOAs (see also Mondor \& Bregman, 1994, who used the same approach in examining the facilitative effect produced by an informative frequency cue [i.e., a frequency sensitivity effect]).

The evidence of location-based and frequency-based auditory IOR obtained by Mondor and Breau (1999) when a judgment orthogonal to the manipulation of cue-target similarity was required established that these phenomena are not the by-product of response biases initiated in reaction to a cue. Whether or not the effects of duration, intensity, and timbre cues apparent in Experiment 1 are also independent of such response biases was evaluated in Experiment 2.

\section{EXPERIMENT 2}

Experiment 2 was essentially identical to Experiment 1, except that the judgment required of listeners was orthogonal to the manipulation of cue-target similarity. Specifically, in all three conditions, 50\% of the targets incorporated a brief silent period. The listeners were required to indicate whether or not the target contained such a gap. As was elaborated above, we instituted this change to determine whether the pattern of performance observed in Experiment 1 could be attributed to response biases initiated in response to the presentation of an advance cue (see, e.g., Kinchla, 1992, for a discussion of this possibility in visual covert orienting). If such biases do dictate the performance pattern, we should find in this experiment little if any effect of the cue on target judgments, because the response required is independent of any information the cue provides. In contrast, if advance duration, intensity, and timbre cues exert their effect through guidance of attentional processes, the performance patterns apparent in Experiment 1 should be replicated.

\section{Method}

\section{Subjects}

Forty-eight undergraduate students attending Mount Allison University volunteered to participate in the experiment in exchange for course credit. Sixteen different listeners participated in each of the duration, intensity, and timbre conditions. None of the listeners reported any hearing impairment.

\section{Materials}

The computer and sound system were identical to those used in Experiment 1. All of the sounds used in Experiment 1 were used again in Experiment 2, plus six new sounds, two for each of the three conditions. Each of these new sounds incorporated a 5-msec silent period. Thus, there were two new sounds for the duration (brief and long), intensity (loud and quiet), and timbre (Sound A and Sound B) conditions. As in Experiment 1, the subjective intensity of all the sounds used within a specific condition were equalized. All other details of sound synthesis were identical to those in Experiment 1.

\section{Design and Procedure}

The sole difference between Experiment 2 and Experiment 1 was that, in all three conditions, listeners were required to judge whether the target contained a brief silent period. The listeners completed 36 practice and 144 experimental trials (24 for each combination of trial type and SOA).

\section{Results}

Notwithstanding the fact that the task used in Experiment 2 was orthogonal to the manipulation of cue-target similarity, the important results of Experiment 1 were 
replicated. Specifically, as is shown in Table 2 and Figure 1 (right panel), performance in the duration, intensity, and timbre conditions followed an IOR pattern in that, in all three conditions, there was a transition from a performance advantage for the repeat trials at the $150-\mathrm{msec}$ SOA to an advantage for the change trials at the $750-\mathrm{msec}$ SOA. These results confirm the existence of duration-, intensity-, and timbre-based auditory IOR and establish that the existence of these phenomena is not founded on response biases generated in reaction to advance cues.

Performance in each condition was examined separately for RT and errors, using two-way within-subjects ANOVAs $(\mathrm{SOA}[150,450$, or $750 \mathrm{msec}] \times$ trial type [repeat or change]). The results of these analyses are described separately below, followed by a comparison of performances in the three conditions.

\section{Duration}

A statistical examination of the RT data collected in the duration condition revealed a significant main effect of SOA $[F(2,30)=18.34, p<.001]$, but not of trial type $[F(1,15)=2.36, p=.14]$. The SOA $\times$ trial type interaction was significant $[F(2,30)=5.83, p<.01]$ because of a transition as a function of SOA from an advantage for the repeat trials at the $150-\mathrm{msec}$ SOA $(p<.001)$ to an advantage for the change trials at the $750-\mathrm{msec}$ SOA $(p<.02)$.

An ANOVA of the error data indicated that the main effect of SOA was significant $[F(2,30)=28.21, p<.001]$, but that neither the main effect of trial type $[F(1,15)=$ $1.10, p=.31]$, nor the trial type $\times$ SOA interaction $[F(2,30)=1.85, p=.17]$, was significant.

\section{Intensity}

A statistical evaluation of the RT data obtained in the intensity condition revealed a significant main effect of SOA $[F(2,30)=15.75, p<.001]$ and a near-significant main effect of trial type $[F(1,15)=3.98, p=.062]$. The interaction between SOA and trial type was also significant $[F(2,30)=6.76, p<.01]$. Planned comparisons indicated that both the advantage for the repeat trials at the 150 -msec SOA $(p<.001)$ and the advantage for the change trials at the $750-\mathrm{msec}$ SOA $(p<.05)$ were significant.
A statistical examination of the error data indicated that whereas the main effect of SOA was significant $[F(2,30)=$ $16.93, p<.001]$, the main effect of trial type and the trial type $\times$ SOA interaction were not $(F<1$ in both cases).

\section{Timbre}

The ANOVA of RTs indicated that whereas the main effect of SOA reached statistical significance $[F(2,30)=$ $18.81, p<.001]$, the main effect of trial type did not $(F<$ 1 ). As was apparent in the analysis of performance in both the duration and the intensity conditions, the interaction between these two variables $[F(2,30)=7.44, p<$ $.01]$ reached significance because a performance advantage for the repeat trials at the 150 -msec SOA $(p<.05)$ reversed to an advantage for the change trials at the 750msec SOA $(p<.02)$.

A complementary analysis of percentage of errors revealed significant main effects of both trial type $[F(1,15)=$ $9.06, p<.01]$ and SOA $[F(2,30)=7.92, p<.01]$. Although the trial type $\times$ SOA interaction was not significant $[F(2,30)=1.84, p=.17]$, planned comparisons documented an advantage for the repeat trials at the 150and $450-\mathrm{msec}$ SOAs ( $p<.05$ in both cases).

\section{Comparison of Performance in the Duration, Intensity, and Timbre Conditions}

A three-way mixed ANOVA including condition (duration, intensity, or timbre $) \times \operatorname{SOA}(150,450$, or $750 \mathrm{msec})$ $\times$ trial type (repeat or change) was performed, using RT as the dependent variable, to determine whether performance differed substantially between conditions. The only effect to reach significance was the main effect of condition $[F(2,81)=4.92, p<.05]$, indicating that mean RT was significantly higher in the intensity condition than in either the duration $(p<.01)$ or timbre $(p<.05)$ conditions. Performance in the duration and timbre conditions did not differ significantly $(p=.35)$. None of the other effects involving the condition variable attained significance $(p>.29)$.

A statistical comparison of the error rates for the three conditions revealed both a significant main effect of condition $[F(2,45)=7.51, p<.01]$ and a significant interaction between condition and SOA $[F(4,90)=6.82, p<$

Table 2

Response Times (RTs), Percentage of Errors (PE), and Standard Errors for the RT Data as a Function of Cue-Target Relation (Duration, Intensity, or Timbre), Trial Type (Repeat or Change), and Stimulus Onset Asynchrony $(150,450$, or $750 \mathrm{msec})$ in Experiment 2

\begin{tabular}{|c|c|c|c|c|c|c|c|c|c|c|}
\hline \multirow{3}{*}{$\begin{array}{c}\text { Cue-Target } \\
\text { Relation }\end{array}$} & \multirow{3}{*}{$\begin{array}{l}\text { Trial } \\
\text { Type }\end{array}$} & \multicolumn{9}{|c|}{ Stimulus Onset Asynchrony } \\
\hline & & \multicolumn{3}{|c|}{$150 \mathrm{msec}$} & \multicolumn{3}{|c|}{$450 \mathrm{msec}$} & \multicolumn{3}{|c|}{$750 \mathrm{msec}$} \\
\hline & & RT & $\mathrm{PE}$ & $S E$ & RT & $\mathrm{PE}$ & $S E$ & RT & $\mathrm{PE}$ & $S E$ \\
\hline \multirow[t]{2}{*}{ Duration } & Repeat & 574 & 23.2 & 18.36 & 506 & 8.8 & 18.41 & 525 & 13.3 & 17.19 \\
\hline & Change & 608 & 25.5 & 25.48 & 525 & 12.5 & 19.18 & 500 & 11.5 & 17.39 \\
\hline \multirow[t]{2}{*}{ Intensity } & Repeat & 666 & 19.2 & 24.89 & 603 & 13.5 & 24.23 & 616 & 12.0 & 25.54 \\
\hline & Change & 714 & 21.9 & 34.15 & 623 & 14.1 & 25.23 & 584 & 10.4 & 20.54 \\
\hline \multirow[t]{2}{*}{ Timbre } & Repeat & 597 & 4.42 & 26.95 & 547 & 2.1 & 23.14 & 557 & 3.6 & 25.69 \\
\hline & Change & 629 & 8.6 & 29.87 & 566 & 4.9 & 25.11 & 517 & 3.6 & 25.37 \\
\hline
\end{tabular}


$.001]$. Whereas the main effect arose because of the lower level of difficulty for the timbre condition than for either the duration or the intensity condition $(p<.01$ for both comparisons), the interaction reflected the fact that this performance advantage for the timbre condition was larger at the 150 -msec SOA than at either the 450 - or the 750 -msec SOA.

\section{Discussion}

The robust evidence of intensity-, timbre-, and durationbased auditory IOR, obtained in this experiment despite the fact that an orthogonal judgment was required provides strong evidence that response biases initiated by the cue are not an important determinant of these phenomena. However, as is shown in Figure 1, the magnitude of the facilitative effects at the 150-msec SOA were substantially larger in Experiment 1 than in Experiment 2. In fact, a direct statistical comparison of performance at the $150-\mathrm{msec}$ SOA in the two experiments supports this inference (108 vs. $60 \mathrm{msec}, p<.001$ ), with no difference in the magnitude of the cuing effect apparent at either the 450 -msec SOA or the 750-msec SOA ( $p>.58$ in both cases). Given that the only procedural difference between the two experiments was in the target judgment required, this asymmetry clearly suggests that the large effects seen in Experiment 1 may be the product of a bias in that experiment in favor of executing a response consistent with the information provided by the cue. Additional support for this conclusion may be drawn from two previous studies in which location- and frequency-based auditory IOR were investigated. Specifically, whereas Mondor and Breau (1999) required listeners to judge the rise-time of the target irrespective of whether the location or the frequency relation between the cue and the target was varied, in one condition reported by Mondor et al. (1998) listeners identified target location when location was varied and target frequency when frequency was varied. A comparison of performances in these two studies reveals that a much smaller average facilitative effect was obtained when the required judgment was independent of the cue-target similarity manipulation (38 vs. $134 \mathrm{msec}$ ).

Interestingly, whereas it appears that the magnitude of the facilitative effect at the 150-msec SOA may be influenced by the opportunity for advance response preparation, this was not true for the inhibitory effect at the 750msec SOA, which did not differ appreciably in the two experiments (the mean advantage for change trials was $30 \mathrm{msec}$ in Experiment 1 and $28 \mathrm{msec}$ in Experiment 2). However, a comparison of the Mondor and Breau (1999) and Mondoret al. (1998) studies reveals a small reduction in the mean advantage for change trials from $36 \mathrm{msec}$ when the required judgment was related to the manipulation of cue-target similarity to $26 \mathrm{msec}$ when it was not. Thus, it is possible that response biases may have a small effect on the magnitude of the inhibitory effect engendered by advance cues. However, we must emphasize that because an orthogonal judgment was used, the results of Experiment 2 establish that the transition in the effect of uninformative duration, intensity, and timbre cues from facilitation at brief SOAs to inhibition at longer SOAs does not depend on response biases.

\section{GENERAL DISCUSSION}

Location and frequency attributes of sounds are often regarded as perceptually dominant over other acoustic features, such as duration, intensity, and timbre (e.g., Bregman, 1990). The temptation is to assume, in consequence, that auditory attention may be guided less well by these subordinate features. The clear evidence of duration-, intensity-, and timbre-based guidance of attention obtained in this study demonstrates that such an assumption is incorrect-at least insofar as stimulus-driven control is concerned. Indeed, that an IOR performance pattern was obtained for all three feature manipulations suggests strongly that this phenomenon is driven by perceptual and cognitive processes that are engaged irrespective of the initiating feature. In documenting the existence of duration-, intensity-, and timbre-based auditory IOR, the present study underscores the utility of this phenomenon for exploring the operation of auditory selective attention.

Any discussion of the fact that auditory IOR may be obtained for several different basic auditory features necessitates a consideration of the extent to which variation in one of these features is independent of changes in the others. For example, there is little doubt that there is a strong relation between the perception of intensity, duration, and pitch so that a physical change in one of these attributes may induce a perceptual change not only in the modified feature, but in the others as well (e.g., Baer, Moore, \& Glasberg, 1999; Hafter \& Carrier, 1972; Moore, 1989; Tekman, 1997; Turnbull, 1944). Similarly, although opinions about the definition of timbre have varied somewhat over the years, with some arguing that timbre is not a distinct perceptual feature (e.g., Ortmann, 1935), general agreement has developed that timbre is an emergent property founded on static and dynamic changes in the relative intensities of the frequencies that make up a complex sound (e.g., Handel, 1989; Hirsh \& Watson, 1996; Iverson, 1995; Iverson \& Krumhansl, 1993; Moore, 1989).

Although perceptions of intensity, duration, timbre, and pitch are, to some extent, interrelated, there are several reasons to conclude that performance in our experiments was driven primarily by the manipulated feature. Specifically, although sound duration may influence perceived intensity, the subjective intensities of all the sounds were equalized in the experiments to ensure that the most salient difference between short and long sounds was in duration, and not in subjective intensity. A second potential concern arises from the fact that timbre was manipulated by differentially weighting the two frequency components that made up the complex sounds used. It is possible that the listeners may have differentiated sounds on the basis of the relative intensities of one of the frequency components, thereby effectively rendering the manipulation one of intensity, rather than one 
of timbre (we thank an anonymous reviewer for pointing this out). However, because the two frequency components were presented from the same spatial position (directly in front of the listener) and were coincident in onset and offset, it is most likely that the complex sound was perceived as a unitary auditory object (see Bregman, 1990 , for a discussion of the factors that influence auditory grouping). Indeed, when individual frequency components are presented concurrently, it is extremely difficult for listeners to identify them even when deliberately trying to do so. Moreover, the success of such an analytic listening strategy appears to depend to a great extent on the availability of contextual information, such as the presentation of a pure tone standard of identical frequency immediately prior to the complex sound (e.g., Bregman \& Pinker, 1978; Helmholtz, 1885/1954). Thus, although it is theoretically possible that timbres were differentiated on the basis of the intensity of a single frequency component, the probability that it was employed by even a small minority of the participants appears to be quite low, given that, under the presentation conditions used, the auditory system acts to integrate the acoustic information likely to have arisen from the same source.

\section{The Dual-Process Model}

Recently, Mondor (1999) offered a theoretical framework consistent with the emerging evidence regarding auditory IOR. This model is founded on the interactions between two separate processes, one that controls the transmission of acoustic information to auditory shortterm memory and the other involved in response assignment. More specifically, Mondor suggests that an attentional template or filter acts to govern the speed with which auditory information is passed on to memory. This template is normally defined by basic auditory features, such as frequency, location, duration, intensity, and timbre. The precise definition of the template varies from one moment to the next as a function of both incoming acoustic information and the listener's current goals. The degree to which the perceptual characteristics of an incoming sound matches the template determines the speed with which it is transmitted to auditory short-term memory. Mondor suggests that in the typical cue-target paradigm, the template may be set in accordance with the properties of the advance cue. Thus, for example, a cue of $50-\mathrm{msec}$ duration and $500 \mathrm{~Hz}$ originating from $45^{\circ} \mathrm{left}$ would set the template for $50 \mathrm{msec}, 500 \mathrm{~Hz}$, and left. If the subsequent target matched these settings (as would occur on a repeat trial), selection would be accomplished quickly. In contrast, if the subsequent target did not match the template, selection would be accomplished more slowly. On the strength of evidence indicating that the magnitude of the facilitative effect of an advance cue apparent at brief SOAs increases as the difference between the cue and the target increases (defined along a single dimension, such as location or frequency), Mondor suggested that selection speed is not dichotomous but varies along a continuum.
Irrespective of whether a target is selected quickly or slowly, eventually a representation of it is passed on to memory, at which point a response assignment process may begin. According to the model, accurate response assignment requires that the memory representation of the target be distinguished from that of the cue. This differentiation process depends on the similarity of the cue and the target representations, with the result that the speed of differentiation increases as the similarity of the two representations decreases (e.g., Ashby \& Perrin, 1988; Hunt, 1995; Luce, 1963). Thus, more time will be required to differentiate cue and target memory representations on trials in which the cue and the target were identical save for time of occurrence (i.e., a repeat trial) than on trials in which the sounds differed in both a fundamental acoustic feature and time of occurrence (i.e., a change trial). According to the framework offered by Mondor (1999), then, whereas the selection processes accomplished at the template engenders an advantage for repeat trials (i.e., a facilitative effect), the memory representation differentiation process involved in response assignment produces an advantage for change trials (i.e., an inhibitory effect).

Mondor (1999) suggests that the time-course of auditory IOR is determined by a tradeoff in the importance of these opposing influences. Given an experimental situation such as the one used in the present study, in which an advance cue is uninformative with respect to the target, a facilitative effect normally is apparent only for quite brief SOAs of a few hundred milliseconds. In recognition of this fact, Mondor suggested that, under these conditions, the specific settings of the parameters of the template dissipate over the course of about 300-400 msec. While active, the modulation of selection speed accomplished at the level of the attentional template normally exerts a larger effect over performance than does the memory representation differentiation process. Once the specific template definition has dissipated, however, the action of the memory process is revealed in a performance advantage for change trials. Thus, although the memory process is active at all SOAs, its contribution to performance is masked at brief SOAs by the countervailing influence of the selection process. In essence, Mondor suggests that the "inhibitory" effect apparent at long SOAs is not indicative of inhibition at all but, rather, reflects the fact that the time required to differentiate two different memory representations depends on similarity.

This framework is consistent not only with the expression of location-, frequency-, duration-, intensity-, and timbre-based auditory IOR, but also with recent experiments demonstrating that the time-course of auditory IOR changes significantly as a function of the predictability of the relation between cue and target. Specifically, Mondor (1999) has shown that whereas an advantage for change trials emerges at about a 700-msec SOA when there is no predictable relation between the cue and the target, it emerges at much longer SOAs when the cue is likely to provide accurate information (i.e., when there is 
a greater proportion of repeat than of change trials) and at briefer SOAs when the cue is likely to provide incorrect information (i.e., when there is a greater proportion of change than of repeat trials). According to the model, this modulation results because of a top-down control process acting to either abbreviate the effective duration of the attentional template when it is likely to impair performance or lengthen it when it is likely to facilitate performance.

The model proposed by Mondor (1999) was designed to account for and explain the known existence and characteristics of auditory IOR. However, one obvious question is whether the model might apply also to visual IOR. Whereas there is an obvious similarity in the time-course and characteristics of location-based IOR for the two modalities of presentation, there are clear differences as well. Specifically, the existence of IOR for nonspatial auditory dimensions, such as duration, frequency, intensity, and timbre, suggests clearly that auditory IOR is a general phenomenon not necessarily linked to spatial location. In contrast, little evidence has been published that visual IOR might arise under conditions in which location is not varied. For example, Law, Pratt, and Abrams (1995) conducted an experiment in which two color patches were presented in succession, with a detection response required to the second of these. They reported a typical IOR performance pattern, with a facilitative effect of the first color patch on detection of the second patch at brief SOAs and an inhibitory effect at longer SOAs. However, Taylor and Klein (1998a) failed to replicate the early facilitative effect and suggested that "the results that Law et al. reported are interesting, but do not demonstrate IOR for color" (p. 1455).

Because it appears that visual IOR may be obtained reliably only for manipulations of spatial position, many have argued that there is a strong relation between activity in the eye movement system and the emergence of visual IOR (e.g., Klein \& MacInnes, 1999; Klein \& Taylor, 1994; Rafal et al., 1989; Sapir, Soroker, Berger, \& Henik, 1999; Taylor \& Klein, 1998b). Similarly, several investigators have suggested that the location-based version of auditory IOR might result because of oculomotor programming directed initially toward and subsequently away from the location of a cue (Reuter-Lorenz \& Rosenquist, 1996; Schmidt, 1996). The demonstration of frequency-, intensity-, timbre-, and duration-based auditory IOR, however, provides strong evidence that such processes are irrelevant with regard to engaging and guiding auditory attention in a variety of contexts. In addition, recent evidence that location-based auditory IOR may be obtained even when eye movements are concurrently planned and executed to visual events strongly suggests that oculomotor processes are not necessary either to guide auditory attention or to produce auditory IOR, even when the location of events is varied (Mondor, Terrio, \& Hurlburt, 2000).

There appear to be obvious differences in the role of spatial location and oculomotor control processes in gen- erating auditory and visual IOR. These differences agree with recent evidence suggesting that auditory and visual selective attention systems are at least partially separate. For example, Mondor and Amirault (1998) showed that advance auditory and visual spatial cues exert stronger facilitative effects over targets of the same modality and suggested that location information could be shared between these systems through endogenous attentional processes (see also, e.g., Spence \& Driver, 1997, and Ward, 1994 , for evidence of generally weaker cross-modal than within-modality exogenous cuing effects).

Notwithstanding the fact that auditory and visual IOR may well be the product of distinct cognitive subsystems, a modified version of the dual-process model proposed by Mondor (1999) could account for many of the known characteristics of visual IOR. Indeed, it would appear that the model need be modified only to recognize (1) the dominance of location in setting the definition of the template and (2) that the oculomotor system is integral in establishing and maintaining template specification. The central components of the model that separate perceptual and memorial processes interact in generating the IOR performance pattern, and that the effective duration of an attentional template may be controlled through a top-down process would remain unchanged in this reformulation. Such a model could explain the existence of visual IOR and the fact that it is largely dependent on variation in spatial location and would be quite consistent with recent evidence reported by Danzinger and Kingstone (1999) that the time-course of visual IOR may be modulated through a change in the predictability of the cue-target relation.

\section{Summary}

The evidence of duration-, intensity-, and timbre-based auditory IOR reported in this paper marks an important contribution to the development of a theoretical account of auditory IOR. These findings establish beyond any doubt that oculomotor control processes, which appear to be of fundamental importance in generating visual IOR, are not generally important in producing auditory IOR. In addition, it appears clear that any basic acoustic feature (or at least any of the five features that have now been studied) may effectively guide the selection of auditory information. Whether differences in the effectiveness of these basic features in engaging attentional processes may emerge when multiple cues are available is not known at this point. However, this important theoretical question is one that we are presently working toward solving.

\section{REFERENCES}

Ashby, F. G., \& Perrin, N. A. (1988). Toward a theory of similarity and recognition. Psychological Review, 95, 124-150.

Baer, T., Moore, B. C. J., \& Glasberg, B. R. (1999). Detection and intensity discrimination of Gaussian-shaped tone pulses as a function of duration. Journal of the Acoustical Society of America, 106, 1907-1916.

Bregman, A. S. (1990). Auditory scene analysis. Cambridge, MA: MIT Press. 
Bregman, A. S., Achim, A., \& Ahad, P. D. (1992). The MAPLE software system. Montreal: McGill University, Department of Psychology.

Bregman, A. S., \& Pinker, S. (1978). Auditory streaming and the building of timbre. Canadian Journal of Psychology, 32, 19-31.

BroAdBENT, D. E. (1957). A mechanical model for human attention and immediate memory. Psychological Review, 64, 205-215.

Broadbent, D. E. (1958). Perception and communication. London: Pergamon.

Carterette, E. C., Friedman, M. P., \& Wyman, M. J. (1966). Feedback and psychophysical variables in signal detection. Journal of the Acoustical Society of America, 39, 1051-1055.

Cherry, E. C. (1953). Some experiments on the recognition of speech, with one and with two ears. Journal of the Acoustical Society of America, 25, 975-979.

Creelman, C. D. (1959). Detection of signals of uncertain frequency. Journal of the Acoustical Society of America, 32, 805-810.

DAI, H., SCHARF, B., \& BUUS, S. (1991). Effective attenuation of signals in noise under focused attention. Journal of the Acoustical Society of America, 89, 2837-2842.

DAnzinger, S., \& Kingstone, A. (1999). Unmasking the inhibition of return phenomenon. Perception \& Psychophysics, 61, 1024-1037.

Green, D. M., McKey, M. J., \& Licklider, J. C. R. (1959). Detection of a pulsed sinusoid in noise as a function of frequency. Journal of the Acoustical Society of America, 31, 1446-1452.

Greenberg, G. Z., \& LARKIN, W. D. (1968). Frequency-response characteristic of auditory observers detecting signals of a single frequency in noise: The probe-signal method. Journal of the Acoustical Society of America, 44, 1513-1523.

HAfTer, E. R., \& CARrier, S. C. (1972). Binaural interaction in lowfrequency stimuli: The inability to trade time and intensity completely. Journal of the Acoustical Society of America, 51, 1852-1862.

HANDEL, S. (1989). Listening: An introduction to the perception of auditory events. Cambridge, MA: MIT Press.

Helmholtz, H. von (1954). On the sensation of tone as a physiological basis for the theory of music (2nd English ed.; A. J. Ellis, Trans.). New York: Dover. (Original work published 1885)

HENKE, W. L. (1990). An interactive dialogue language for time signal processing. Cambridge, MA: MIT, Research Laboratory of Electronics.

Hirsh, I. J. \& WAtson, C. S. (1996). Auditory psychophysics and perception. Annual Review of Psychology, 47, 461-484.

Huggins, W. H. (1952). A phase principle for complex frequency analysis and its implications in auditory theory. Journal of the Acoustical Society of America, 24, 582-589.

Hunt, R. R. (1995). The subtlety of distinctiveness: What von Restorff really did. Psychonomic Bulletin \& Review, 2, 105-112.

IvERSON, P. (1995). Auditory stream segregation by musical timbre: Effects of static and dynamic acoustic attributes. Journal of Experimental Psychology: Human Perception \& Performance, 21, 751-763.

Iverson, P., \& KrumhansL, C. L. (1993). Isolating the dynamic attributes of musical timbre. Journal of the Acoustical Society of America, 94, 2595-2603.

JEFFRESS, L. A. (1967). Stimulus-oriented approach to detection reexamined. Journal of the Acoustical Society of America, 41, 480-488.

Johnson, D. M., \& HAFTER, E. R. (1980). Uncertain-frequency detection: Cuing and condition of observation. Perception \& Psychophysics, 28, 143-149.

Johnston, W. A., \& Heinz, S. P. (1978). Flexibility and capacity demands of attention. Journal of Experimental Psychology: General, 107, 420-435.

Kinchla, R. A. (1992). Attention. Annual Review of Psychology, 43, 711-742.

KLeIN, R. M., \& MacInnes, W. J. (1999). Inhibition of return is a foraging facilitator in visual search. Psychological Science, 10, 346-352.

Klein, R. M., \& TAYlor, T. L. (1994). Categories of cognitive inhibition with reference to attention. In D. Dagenbach \& T. H. Carr (Eds.), Inhibitory processes in attention, memory, and language (pp. 113150). San Diego: Academic Press.

LaW, M. B., Pratt, J., \& Abrams, R. A. (1995). Color-based inhibition of return. Perception \& Psychophysics, 57, 401-408.

Luce, R. D. (1963). Detection and recognition. In R. D. Luce, R. R.
Bush, \& E. Galanter (Eds.), Handbook of mathematical psychology (Vol. 1, pp. 103-190). New York: Wiley.

Macmillan, N. A., \& Schwartz, M. (1975). A probe-signal investigation of uncertain-frequency detection. Journal of the Acoustical Society of America, 58, 1051-1058.

MAYlOR, A. M., \& Hockey, R. (1985). Inhibitory component of externally controlled covert orienting in space. Journal of Experimental Psychology: Human Perception \& Performance, 11, 777-787.

Mondor, T. A. (1999). Predictability of the cue-target relation and the time-course of auditory inhibition of return. Perception \& Psychophysics, 61, 1501-1509.

Mondor, T. A., \& Amirault, K. J. (1998). Effect of same- and differentmodality spatial cues on auditory and visual target identification. Journal of Experimental Psychology: Human Perception \& Performance, 24, 745-755.

Mondor, T. A., \& BreaU, L. M. (1999). Facilitative and inhibitory effects of location and frequency cues: Evidence of a modulation in perceptual sensitivity. Perception \& Psychophysics, 61, 438-444.

Mondor, T. A., Breau, L. M., \& Milliken, B. (1998). Inhibitory processes in auditory selective attention: Evidence of location-based and frequency-based inhibition of return. Perception \& Psychophysics, 60, 296-302.

Mondor, T. A., \& Bregman, A. S. (1994). Allocating attention to frequency regions. Perception \& Psychophysics, 56, 268-276.

Mondor, T. A., Terrio, N. A., \& Hurlburt, J. (2000). On the role of eye movements and voluntary saccade preparation in generating location-based auditory inhibition of return. Canadian Journal of Experimental Psychology, 54, 326-338.

Moore, B. C. J. (1989). An introduction to the psychology of hearing. San Diego: Academic Press.

Moray, N. (1972). Listening and attention. Baltimore: Penguin Books.

Moray, N. (1974). A data base for theories of selective listening. In P. M. A. Rabbitt \& S. Dornic (Eds.), Attention and performance $V$ (pp. 119-135). New York: Academic Press.

Moray, N., Fitter, M., Ostry, D., Favreau, D., \& Nagy, V. (1976). Attention to pure tones. Quarterly Journal of Experimental Psychology, 28, 271-283.

Ortmann, O. (1935). What is tone-quality? Musical Quarterly, 21, 442-450.

Penner, M. J. (1972). The effect of payoffs and cue tones on detection of sinusoids of uncertain frequency. Perception \& Psychophysics, 11, 198-202.

Posner, M. I., \& Cohen, Y. (1984). Components of visual orientating. In H. Bouma \& D. G. Bouwhuis (Eds.), Attention and performance X: Control of language processes (pp. 531-555). Hillsdale, NJ: Erlbaum.

Rafal, R. D., Calabressi, P. A., Brennan, C. W., \& Sciolto, T. K. (1989). Saccade preparation inhibits reorienting to recently attended locations. Journal of Experimental Psychology: Human Perception \& Performance, 15, 673-685.

Reuter-Lorenz, P. A., Jha, A. P., \& Rosenquist, J. N. (1996). What is inhibited in inhibition of return? Journal of Experimental Psychology: Human Perception \& Performance, 22, 367-378.

Reuter-Lorenz,P. A., \& Rosenquist, J. N. (1996). Auditory cues and inhibition of return: The importance of oculomotor activation. Experimental Brain Research, 112, 119-126.

Sapir, A., Soroker, N., Berger, A., \& Henik, A. (1999). Inhibition of return in spatial attention: Direct evidence for collicular generation. Nature Neuroscience, 2, 1053-1054.

Scharf, B., Quigley, S., Aoki, C., Peachey, N., \& Reeves, A. (1987). Focused auditory attention and frequency sensitivity. Perception \& Psychophysics, 42, 215-223.

Schlauch, R. S., \& Hafter, E. R. (1991). Listening bandwidths and frequency uncertainty in pure-tone signal detection. Journal of the Acoustical Society of America, 90, 1332-1339.

ScHmidT, W. C. (1996). Inhibition of return without visual input. Neuropsychologia, 34, 943-952.

Shaw, M. L. (1978). A capacity allocation model for reaction time. Journal of Experimental Psychology: Human Perception \& Performance, 4, 586-598.

Shaw, M. L. (1984). Division of attention among spatial locations: A 
fundamental difference between detection of letters and detection of luminance increments. In H. Bouma \& D. G. Bouwhuis (Eds.), Attention and performance $X$ : Control of language processes (pp. 109121). Hillsdale, NJ: Erlbaum.

Sorkin, R. D., Pastore, R. E., \& Gilliom, J. D. (1968). Signal probability and the listening band. Perception \& Psychophysics, 4, 10-12.

Spence, C., \& Driver, J. (1997). Audiovisual links in exogenous covert spatial orienting. Perception \& Psychophysics, 59, 1-22.

Spence, C., \& Driver, J. (1998). Auditory and audiovisual inhibition of return. Perception \& Psychophysics, 60, 125-139.

Swets, J. A. (1963). Central factors in auditory frequency selectivity. Psychological Bulletin, 60, 429-440.

TAYLOR, T. L., \& KleIN, R. M. (1998a). Inhibition of return to color: A replication and nonextension of Law, Pratt, and Abrams (1995). Perception \& Psychophysics, 60, 1452-1456.

TAY LOR, T. L., \& KLEIN, R. M. (1998b). On the causes and effects of inhibition of return. Psychonomic Bulletin \& Review, 5, 625-643.

TeKman, H. G. (1997). Interactions of perceived intensity, duration, and pitch in pure tone sequences. Music Perception, 14, 281-294.

Tipper, S. P., Weaver, B., Jerreat, L. M., \& Burak, A. L. (1994). Object-based and environment-based inhibition of return of visual attention. Journal of Experimental Psychology: Human Perception \& Performance, 20, 478-499.

Treisman, A. M. (1960). Contextual cues in selective listening. Quarterly Journal of Experimental Psychology, 12, 242-248.

Treisman, A. M. (1969). Strategies and models of selective attention. Psychological Review, 76, 282-299.

Treisman, A. M. (1972). Detection of binaural tone stimuli: Time sharing or criterion change? Journal of the Acoustical Society of America, 51, 625-631.
Turnbull, W. W. (1944). Pitch discrimination as a function of tonal duration. Journal of Experimental Psychology, 34, 302-316.

WARD, L. M. (1994). Supramodal and modality-specific mechanisms for stimulus-driven shifts of auditory and visual attention. Canadian Journal of Experimental Psychology, 48, 242-259.

\section{NOTES}

1. The phrase location-based auditory IOR is used in this paper to refer to the transition in performance from facilitation to inhibition as a function of SOA that may occur when the location relation between cue and target is manipulated. Similarly, the phrases frequency-based, intensity-based, timbre-based, and duration-based IOR refer to the same transition that may arise when the duration, frequency, intensity, and timbre relation between cue and target are manipulated.

2. In contrast with discrimination of sounds on the basis of pitch or timbre, discrimination on the basis of duration cannot begin at the onset of the sound. To correct for this constraint, for the duration condition, RT was measured from the end of each sound. Because, in both Experiments 1 and 2, there were equal numbers of short and long sounds for each combination of SOA and trial type, any difference between short and long sounds in the time required to identify duration (Experiment 1) or detect the presence of a gap (Experiment 2) could not have systematically influenced the results obtained.

(Manuscript received October 26, 1999; revision accepted for publication August 18, 2000.) 